\title{
STRONG BOEHMIANS
}

\author{
ELLEN R. DILL AND PIOTR MIKUSIŃSKI
}

(Communicated by J. Marshall Ash)

\begin{abstract}
A new class of generalized functions is introduced. The objects are defined as convolution quotients. The space is larger than the space of Schwartz distributions but smaller than the space of Boehmians.
\end{abstract}

\section{INTRODUCTION}

Boehmians were introduced in [6] as a generalization of both regular Mikusiński operators [3] and Schwartz distributions. Among the advantages of regular operators are the algebraic character of the construction and existence of regular operators of infinite order with compact support. On the other hand, regular operators, as all Mikusiński operators, have the support bounded from the left. Boehmians have an algebraic character of Mikusiński operators and at the same time do not have any restriction on the support. They contain all Schwartz distributions, Roumieu ultradistributions, regular operators, and other new objects $[8,12]$. In particular, the series $\sum_{n=-\infty}^{\infty} C_{n} e^{i 2^{n} t}$ converges for any sequence of coefficients $C_{n}$. These sequences can be used to construct new solutions of the Laplace equation $[4,9]$. Those results may suggest that the space of Boehmians is too large.

In this note we define a new space of generalized functions which still has the algebraic character of Boehmians, contains all Schwartz distributions, but does not contain sums of trigonometric series with coefficients that grow too fast.

The definition of Boehmians can be found in [4, 6-9]. Sections 2 and 3 of this note contain some results from [5].

\section{STRONG BoEHMians}

Let $\mathscr{E}$ be a Banach space, $\mathfrak{N}$ the space of all infinitely differentiable functions from $[1, \infty) \times \mathbb{R}^{N}$ into $\mathscr{E}$, and $\mathfrak{F}$ the space of all real-valued infinitely differentiable functions $\phi$ with compact support in $\mathbb{R}^{N}$ such that $\int \phi=1$. By * we denote the operation of convolution over $\mathbb{R}^{N}$. In particular, if $f \in \mathfrak{N}$ and $\phi \in \mathfrak{F}$, then

$$
(f * \phi)(x)=\{f(\alpha, x)\} *\{\phi(x)\}=\int_{\mathbb{R}^{N}} f(\alpha, v) \phi(x-v) d v .
$$

Received by the editors September 11, 1991 and, in revised form, March 16, 1992. 1991 Mathematics Subject Classification. Primary 44A40; Secondary 46F05, 44A35. Key words and phrases. Boehmian, convolution quotient, Schwartz distributions. 
Let $\mathfrak{A}$ be the collection of all pairs $(f, \phi)$ such that $f \in \mathfrak{N}, \phi \in \mathfrak{F}$, and $\{f(\alpha, x)\} *\left\{\beta^{N} \phi(\beta x)\right\}=\{f(\beta, x)\} *\left\{\alpha^{N} \phi(\alpha x)\right\}$ for all $\alpha, \beta \in[1, \infty)$. If $(f, \phi),(g, \psi) \in \mathfrak{A}$ and $\{f(\alpha, x)\} *\left\{\beta^{N} \psi(\beta x)\right\}=\{g(\beta, x)\} *\left\{\alpha^{N} \phi(\alpha x)\right\}$ for all $\alpha, \beta \in[1, \infty)$, then we write $(f, \phi) \sim(g, \psi)$. It can easily be proved that $\sim$ is an equivalence. By $\mathfrak{S}\left(\mathbb{R}^{N}, \mathscr{E}\right)$, or simply $\mathfrak{S}$, we denote the space of equivalence classes in $\mathfrak{A}$. Elements of $\mathfrak{S}$ are called strong Boehmians.

It will be convenient to use the notation $d_{\alpha} \phi(x)=\alpha^{N} \phi(\alpha x), \alpha \geq 1$.

2.1. If $\phi, \psi \in \mathfrak{F}$, then $\phi * \psi \in \mathfrak{F}$.

2.2. If $f \in \mathfrak{N}$ and $\phi \in \mathfrak{F}$, then $f * \phi \in \mathfrak{N}$.

2.3. If $(f, \phi) \in \mathfrak{A}$ and $\psi \in \mathfrak{F}$, then $(f * \psi, \phi * \psi) \in \mathfrak{A}$ and $(f, \phi) \sim$ $(f * \psi, \phi * \psi)$.

2.4. If $\phi \in \mathfrak{F}$ and $\alpha \geq 1$, then $d_{\alpha} \phi \in \mathfrak{F}$.

2.5. Let $(f, \phi) \in \mathfrak{A}$ and $\omega>0$. If $g(\alpha, x)=f(\alpha+\omega, x)$ and $\psi=d_{\omega} \phi$, then $(g, \psi) \in \mathfrak{A}$ and $(g, \psi) \sim(f, \phi)$.

If $F \in \mathfrak{S}$ and $F=(f, \phi)$, we will write $F=f / \phi$, which is not quite correct but will not lead to any misunderstanding. We adopt the following definitions:

$$
\lambda \frac{f}{\phi}=\frac{\lambda f}{\phi}, \quad \frac{f}{\phi}+\frac{g}{\psi}=\frac{f * \psi+g * \phi}{\phi * \psi}, \quad \frac{f}{\phi} * \psi=\frac{f * \psi}{\phi},
$$

where $f, g \in \mathfrak{N}, \phi, \psi \in \mathfrak{F}$, and $\lambda \in \mathbb{R}$. It is easy to show that the above operations are well defined and that $\mathfrak{S}$ is a vector space. In the following theorem we prove that any infinite sequence of strong Boehmians can be represented with a common denominator. In the proof we use infinite convolutions [2].

2.6. Theorem. If $F_{1}, F_{2}, \ldots \in \mathfrak{S}$, then there exist $f_{1}, f_{2}, \ldots \in \mathfrak{N}$ and $\phi \in \mathfrak{F}$ such that $F_{n}=f_{n} / \phi$ for all $n \in \mathbb{N}$.

Proof. For $n=1,2, \ldots$, let $F_{n}=g_{n} / \psi_{n}$ and let $\omega_{n} \geq 1$ be such that $d_{\omega_{n}} \psi_{n}(x)=0$ for $\|x\| \geq 2^{-n}$. Then the infinite convolutions

$$
\phi=d_{\omega_{1}} \psi_{1} * d_{\omega_{2}} \psi_{2} * \cdots
$$

and

$$
\gamma_{n}=d_{\omega_{1}} \psi_{1} * d_{\omega_{2}} \psi_{2} * \cdots * d_{\omega_{n-1}} \psi_{n-1} * d_{\omega_{n+1}} \psi_{n+1} * \cdots
$$

belong to $\mathfrak{F}$ (see [7]). Now define $h_{n}(\alpha, x)=g_{n}\left(\alpha+\omega_{n}, x\right)$. Then

$$
F_{n}=\frac{g_{n}}{\psi_{n}}=\frac{h_{n}}{d_{\omega_{n}} \psi_{n}}=\frac{h_{n} * \gamma_{n}}{d_{\omega_{n}} \psi_{n} * \gamma_{n}}=\frac{h_{n} * \gamma_{n}}{\phi}
$$

by 2.3 and 2.5 .

A sequence of strong Boehmians $\left\{F_{n}\right\}$ is said to converge to a strong Boehmian $F$ if $F=f / \phi$ and $F_{n}=f_{n} / \phi$, for some $f, f_{n} \in \mathfrak{N}$ and $\phi \in \mathfrak{F}$, and $f_{n} \rightarrow f$ uniformly on compact subsets of $[1, \infty) \times \mathbb{R}^{N}$.

Let $D^{p}=\left(\partial / \partial x_{1}\right)^{p_{1}} \ldots\left(\partial / \partial x_{N}\right)^{p_{N}}$, where $p=\left(p_{1}, \ldots, p_{N}\right)$ and $p_{1}, \ldots, p_{N}$ are nonnegative integers. For $f / \phi \in \mathfrak{S}$, define $D^{p}(f / \phi)=\left(D^{p} f\right) / \phi$. It can easily be proved that $D^{p}$ is a well-defined operation on $\mathfrak{S}$.

\section{S AND SCHWARTZ DISTRIBUTIONS}

In this section we show that the space of $\mathscr{E}$-valued distributions $[\mathscr{D}(\mathbb{R}), \mathscr{E}]$ (see [14]) can be identified with a proper subspace of $\mathfrak{S}$. 
3.1. Theorem. Let $\phi \in \mathfrak{F}$. The mapping $\mathscr{I}_{1}:[\mathscr{D}(\mathbb{R}), \mathscr{E}] \rightarrow \mathfrak{S}$ defined by

$$
\mathscr{I}_{1}(f)=\frac{f * d_{\alpha} \phi}{\phi}
$$

is a continuous injection which is independent of $\phi$.

Proof. To prove that $\mathscr{I}_{1}$ is well defined and independent of $\phi$ we use the following property of $\mathscr{E}$-valued distributions (see [14, Theorem 3.4-1]): If $f \in$ $[\mathscr{D}(\mathbb{R}), \mathscr{E}]$ and $K$ is a compact interval in $\mathbb{R}^{N}$, then there exist a continuous $\mathscr{E}$-valued function $g$ on $\mathbb{R}^{N}$ and a multi-index $p$ such that

$$
\langle f, \psi\rangle=\int_{\mathbb{R}^{N}} g(v) D^{p} \psi(v) d v
$$

for every test function $\psi$ with support in $K$. Consequently, locally we have $f * \phi=(-1)^{|p|} g * D^{p} \phi$, and thus $\left(f * d_{\alpha} \phi_{1}\right) * d_{\beta} \phi_{2}=\left(f * d_{\beta}=\phi_{2}\right) * d_{\alpha} \phi_{1}$ for all $\phi_{1}, \phi_{2} \in \mathfrak{F}$ and $\alpha, \beta \geq 1$.

To prove continuity of $\mathscr{I}_{1}$ note that, for every compact interval $K \subset \mathbb{R}^{N}$ and $\left[1, \alpha_{1}\right] \subset \mathbb{R}$, the set $\left\{d_{\alpha} \phi(\cdot-v): v \in K, \alpha \in\left[1, \alpha_{1}\right]\right\}$ is a compact set in the space of test functions and then use the Banach-Steinhaus theorem.

Now we construct a strong Boehmian in $\mathfrak{S}(\mathbb{R}, C)$ which is not a distribution. It is a modification of the example given by Boehme in [3]. We use the notation $f(\alpha, t)$ instead of $f(\alpha, x)$ to emphasize the fact that the dimension $N=1$. The example can be extended to $\mathbb{R}^{N}$ without difficulty.

3.2. Example. From the Denjoy-Carleman theorem (see, e.g., [13]) it follows that there exists $\phi \in \mathfrak{F}$ such that

$$
\left|\frac{d^{n}}{d t^{n}} \phi^{(n)}(t)\right| \leq A B^{n}(2 n) \text { ! for all } t \in \mathbb{R} .
$$

Define

$$
f(\alpha, t)=\sum_{n=0}^{\infty} \frac{d^{n}\left(d_{\alpha} \phi(t)\right) / d t^{n}}{(3 n) !} .
$$

One can easily show that the series converges uniformly on compact subsets of $[1, \infty) \times \mathbb{R}$ and that $F=f / \phi$ is a strong Boehmian which is not a distribution.

\section{4. $\mathfrak{S}$ IS A PROPER SUBSPACE OF THE SPACE OF BOEHMIANS}

The space $\mathfrak{B}$ of Boehmians is a complete metrizable space with respect to the so-called $\Delta$-convergence [7]. In the following theorem we refer to that convergence.

4.1. Theorem. The mapping $\mathscr{I}_{2}: \mathfrak{S} \rightarrow \mathfrak{B}$ defined by

$$
\mathscr{I}_{2}\left(\frac{f}{\phi}\right)=\frac{f(n, \cdot)}{d_{n} \phi}
$$

is a continuous injection which is independent of $\phi$.

Proof. Clearly, $\mathscr{I}_{2}$ is independent of $\phi$. Suppose now that $F_{n}=f_{n} / \phi, F=$ $f / \phi$, and $f_{n} \rightarrow f$ uniformly on compact subsets of $[1, \infty) \times \mathbb{R}^{N}$. Define $\phi_{k}=d_{k} \phi$. Then $F_{n} * \phi_{k}=\left\{f_{n}(k, x)\right\} \rightarrow\{f(k, x)\}=F * \phi_{k}$, which implies $F_{n} \rightarrow F$ in $\mathfrak{B}$. 
To show that $\mathfrak{S}$ is a proper subspace of $\mathfrak{B}$ we use Fourier coefficients of periodic Boehmians. Growth of Fourier coefficients of Boehmians has been studied by Nemzer in [10-12]. Since the essential ideas are well exhibited in case $N=1$, we only give an example in that case.

4.2. Example. It can be shown that $\sum_{n=0}^{\infty} C_{n} e^{i 2^{n} t}$ is a Bohemian for an arbitrary sequence $C_{n} \in \mathbb{R}$. We will show that it is not true for strong Boehmians. For example, $\sum_{n=0}^{\infty} e^{5^{n}} e^{i 2^{n} t}$ is not a strong Boehmian. Indeed, if $\phi \in \mathfrak{F}$, then

$$
\left\{\sum_{n=0}^{\infty} e^{5^{n}} e^{i 2^{n} t}\right\} *\left\{d_{\alpha} \phi(t)\right\}=\sum_{n=0}^{\infty} e^{5^{n}} \hat{\phi}\left(\frac{2^{n}}{\alpha}\right) e^{i 2^{n} t}
$$

where $\hat{\phi}$ is the Fourier transform of $\phi$. By the Paley-Wiener theorem [1, 6.8.1], $\hat{\phi}$ is an entire function of exponential type. Consequently [1, 5.4.2]

$$
\left|\hat{\phi}\left(t_{n}\right)\right| \geq e^{-t_{n}^{2}}
$$

for some $t_{n} \rightarrow \infty$. Now there exist increasing sequences of positive integers $\left\{q_{n}\right\}$ and $\left\{k_{n}\right\}$, and $\alpha_{n} \in[1,2]$ such that $t_{q_{n}}=2^{k_{n}} / \alpha_{n}$. Then

$$
\left|\hat{\phi}\left(t_{q_{n}}\right)\right| \geq e^{-t_{q_{n}}^{2}}=e^{-4^{k_{n}} / \alpha_{n}^{2}} \geq e^{-4^{k_{n}}}
$$

and thus

$$
\left|e^{5^{k_{n}}} \hat{\phi}\left(\frac{2^{k_{n}}}{\alpha_{n}}\right)\right| \geq e^{5^{k_{n}}} e^{-4^{k_{n}}} \rightarrow \infty .
$$

Therefore, the series $\sum_{n=0}^{\infty} e^{5^{n}} \hat{\phi}\left(2^{n} / \alpha\right) e^{i 2^{n} t}$ cannot converge uniformly on any set containing $[1,2] \times\{0\}$ for any $\phi \in \mathfrak{F}$.

\section{REFERENCES}

1. R. P. Boas, Entire functions, Academic Press, New York, 1954.

2. T. K. Boehme, On sequences of continuous functions and convolution, Studia Math. 25 (1965), 333-335.

3. - The support of Mikusinski operators, Trans. Amer. Math. Soc. 176 (1973), 319-334.

4. J. Burzyk, Nonharmonic solutions of the Laplace equation, Generalized Functions, Convergence Structures and their Applications, Plenum Press, New York and London, 1988, pp. 3-11.

5. E. R. Dill, Strong Boehmians, research report, Dept. of Math., Univ. of Central Florida, 1991.

6. J. Mikusiński and P. Mikusiński, Quotients de suites et leurs applications dans l'analyse fonctionnelle, C. R. Acad. Sci. Paris Sér. I Math. 293 (1981), 463-464.

7. P. Mikusiński, Convergence of Boehmians, Japan. J. Math. (N.S.) 9 (1983), 159-179.

8. __ Boehmians and generalized functions, Acta Math. Hungar. 51 (1988), 271-281.

9. __ On harmonic Boehmians, Proc. Amer. Math. Soc. 106 (1989), 447-449.

10. D. Nemzer, Periodic Boehmians, Internat. J. Math. Math. Sci. 12 (1989), 685-692.

11. __ Periodic generalized functions, Rocky Mountain J. Math. 20 (1990).

12. __ Periodic Boehmians. II, Bull. Austrian Math. Soc. 44 (1991), 271-278.

13. W. Rudin, Real and complex analysis, 2nd ed., McGraw-Hill, New York, 1974.

14. A. H. Zemanian, Realizability theory for continuous linear systems, Academic Press, New York, 1972.

Department of Mathematics, University of Central Florida, Orlando, Florida 32816 